\title{
Endodontic Microsurgical Instruments - A Review
}

\author{
Prathap M.S. ${ }^{1}$, Reshma Pradeep. ${ }^{2}$ \\ 1, 2 Department of Conservative Dentistry and Endodontics, Yenepoya Dental College, Yenepoya \\ (Deemed to Be University), Deralakatte, Mangalore, Karnataka, India.
}

\section{ABSTRACT}

Indications for endodontic surgery include failed nonsurgical treatment or retreatment, anatomical problems or iatrogenic errors. Endodontic surgery was considered as the last option with instruments that were unsuitable, surgical sites with inadequate vision and increased incidence of post-operative complication. But today endodontic surgery has evolved into endodontic microsurgery with the advancement in illumination, magnification, instruments and materials. Higher magnification and micro-instruments have increased the clinical outcome of endodontic surgery. Traditional instruments used in endodontic surgery are too large for the small osteotomy sites of microsurgery. Few of the instruments used for microsurgery are the smaller version of the traditional instruments. Endodontic microsurgery represents a minimally invasive treatment option with predictable outcome with the use of micro-instruments. A high success rate of nearly $93.5 \%$ is reported, thus, making microsurgery a predictable option for the management of periapical pathology where surgical root treatment is not possible. The advanced techniques developed to overcome the barriers seen in traditional endodontic surgery have allowed the clinicians to achieve higher success rates. Along with the micro-instruments, the introduction of biomimetic materials has also led to the favourable results after endodontic surgery. This article discusses the different microsurgical instruments available for microsurgical procedures.

\section{KEY WORDS}

Curette, Microblade, Microsurgery, Piezotomes, Retractor, Ultrasonic.
Corresponding Author: Dr. Reshma Pradeep, 2102 Block A, Maurishka Palace, Kadri Kambla Road, Kadri, Mangalore - 575003, Karnataka, India. E-mail: drreshmapradeep@gmail.com

DOI: $10.14260 / j e m d s / 2021 / 320$

How to Cite This Article:

Prathap MS, Pradeep $R$. Endodontic microsurgical instruments - a review. J Evolution Med Dent Sci 2021;10(20):15321538, DOI: $10.14260 /$ jemds/2021/320

Submission 19-11-2020,

Peer Review 14-03-2021,

Acceptance 20-03-2021,

Published 17-05-2021.

Copyright (c) 2021 Prathap M.S. et al. This is an open access article distributed under Creative Commons Attribution License [Attribution 4.0 International (CC BY 4.0)] 


\section{BACKGROUND}

Treatment of lesions of endodontic origin which usually do not respond or not possible to treat by conventional endodontic therapy leaves us with the option of surgical endodontics. ${ }^{1}$ Elimination of any extra radicular infection and foreign bodies along with removal of lesions like persistent apical granulomas and cysts is the fundamental objective of surgical endodontic treatment. ${ }^{2}$ Traditional endodontic surgery techniques have significantly developed during the past two decades and has emerged as a preferred treatment option to maintain the natural dentition which is predictable. ${ }^{3}$ Utilisation of the operating microscope, microsurgical instruments, better hard and soft tissue management and use of more biologically acceptable root-end filling materials are few of the modern techniques involved. ${ }^{3}$

Surgical procedure on very small and complex structures with an operating microscope defines microsurgery. ${ }^{4,5,6}$ Endodontic microsurgery provides the option of minimal invasive treatment by allowing the retention of the existing intact coronal restoration, and maintaining the gingival and periodontal structures from a functional and aesthetic aspect, in comparison to traditional nonsurgical retreatment or implant placement after complete extraction. ${ }^{7}$

Working with higher magnifications cannot be achieved by traditional surgical instruments as they are too large. ${ }^{8}$ Endodontic microsurgery is carried out by specifically designed microsurgical instruments to achieve the required precision. ${ }^{9}$ Microsurgical procedure encompasses triad of magnification, illumination and instruments.5,8 This article discusses the various microsurgical instruments currently available and their application in microsurgery. First generation micro instruments were designed and manufactured by Dr. Garry Carr. ${ }^{8}$

\section{MICROSURGICAL INSTRUMENTS}

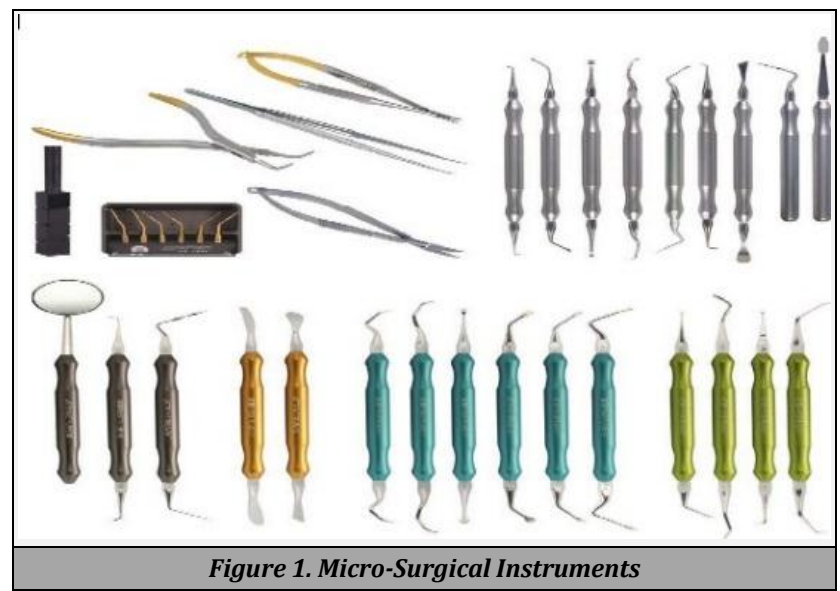

Endodontic microsurgery procedure involves the magnification and illumination by the microscope, which is complimented with micro instruments. ${ }^{10}$ Microsurgical instruments are classified into following groups. ${ }^{5}$

- Examination and inspection.

- Incision, elevation and curettage.

- Retraction.

- Osteotomy and root end resection.
- Root end preparation.

- Retro-fill instruments.

- Irrigation.

- $\quad$ Suturing.

\section{Micromirrors}

Highly polished stainless steel is used to manufacture doublesided mirrors and is available in the market in varying sizes and shapes from round or rectangular shapes, which afford ideal access and visibility in all clinical situations. A crucial feature of the mirror neck is its flexibility. Entire resected root surface can be reflected using a rectangular micromirror positioned at a $45^{\circ}$. The resected root could not have been viewed completely and clearly without the flexibility to bend the micro mirror neck to accommodate the angle. Rectangular micromirrors are available in variable width of $2 \mathrm{~mm}, 3 \mathrm{~mm}$, and $4 \mathrm{~mm}$ on a flexible stainless handle and hence, becomes the perfect choice for use as round mirrors have restricted usage on resected root surface of central incisor. ${ }^{9,11}$

\section{Microexplorer}

Mircroexplorer is a double ended instrument with one end having 90 degree bent with a $2 \mathrm{~mm}$ tip and 130 degrees on the other which makes it extremely useful for identifying areas of leakage on the resected root surface and also to distinguish a fracture line / craze line. ${ }^{9}$ Hence, for sounding the cortical plate and locating the apex, microexplorers are ideal. ${ }^{12}$

\section{INCISION, ELEVATION AND CURETTAGE INSTRUMENTS}

\section{C Blade and Handle}

They are small enough to manage the interproximal papilla, however, large enough so as to create a vertical releasing incision that too in one stroke and hence, it becomes the ideal scalpel blade of choice. ${ }^{9}$

\section{Micro Blade}

If the interproximal spaces are tight and indicated in the aesthetic zone or in the presence of poorly keratinised tissue, then the micro blades are useful.9,13

\section{Elevators}

Design of elevators is such that elevation of the gingiva from the underlying cortical bone is performed with minimal trauma to the tissue. A thin, sharp, triangular beak on one end and a sharp, rounded beak that varies in size on the other end are its salient features of these elevators. This has thin edges and points to allow the precise and complete soft tissue elevation with minimum trauma. ${ }^{9}$

\section{Prichard PPR3}

Designed for separating \& retracting soft tissue away from bone. ${ }^{8}$ 


\section{MAR Curette}

MAR curettes developed by professor Marwan Abou Ras are used for atraumatic elevation of endodontic and periodontic flaps. For thin eroded bone over pathologically involved roots, scraping thin cortical bone and exposing apices of fenestrated roots, straight curettes are used. These straight curettes provide continuous tip visibility of palatal root tips, lingual, far distal anatomies. ${ }^{14}$

Angled MAR curettes are available as -

- $\quad$ MAR-C1 Long shank and sharp tip are perfect to remove diseased hard or soft tissue which are trapped in the smallest bone.

- Full mucoperiosteal flap elevation of maxillary lateral incisors and mandibular lower anterior, MAR-C2 is used.

- MAR-C3 is the most universal mucoperiosteal flap elevation instrument. The long shank and sharp tip form atraumatic, controlled flaps on gingival crevices of all posterior teeth centrals, preparing gingival sulci spaces for the MAR-C4.

- MAR-C4 used for surgical curettage of huge osseous alveolar defects and palatal flap elevation or for initiating attached gingival elevation prior to using the Allen P9A periosteal.

Straight MAR curettes are available as:

- MAR-SC1 are the smallest straight curette that ensures perfect straight-line access and control, mainly during a buccal approach to the palatal roots of the maxillary first premolars and molars.

- MAR-SC2 are preferable for removing buccal cortical plate defects and root exposure.

- $\quad$ MAR-SC3 excellent for removing fractured root fragments and debriding palate defects.

- $\quad$ MAR-SC4 is the largest straight curette, used for debriding large defects and scraping diseased bone.

\section{Molts Curette No 2 / 4}

Molt \#2 - 4, these are a combination of \#2 and \#4 Molt Curette Tips of $3.3 \mathrm{~mm}$ and $7 \mathrm{~mm}$ widths on the ends. This curette is used for curettage, cyst removal, and debridement of tooth socket. ${ }^{5}$

\section{Mini Curette and Mini Molt Curette}

Curettage instruments include mini endodontic curettes, surgical curettes, and periodontal curettes and are utilised for a microsurgical procedure. ${ }^{9}$ Micro-curettes are used for winking out root tips and elevating soft tissue from the cavity.., 12

\section{Buser Periosteal Elevator}

These elevators are used for reflecting and retracting the mucoperiosteum. It has a sharp round blade and narrow pointed end for interproximal use, which for enriched contrast between the instrument, underlying tissue and the surgical site has distinct black finish. A matte finished handle and black working ends reduces the reflection of light.12,15

\section{Endodontic Curette}

Sharp dissection of the periosteal tissue without tissue damage can be made with ruddle curettes. Undermining elevation of the flap with these small curettes along with micro-surgical incision comprises the two primary steps in automatic flap management. 16

\section{Columbia No. 13 / 14 Curette}

Columbia \#13 and \#14 curettes allow access to the hardest part to reach the lingual aspect of the root, due to the gradual curve in them. ${ }^{17}$ Curettage generally is not microsurgical procedure; however, any periodontal curette can be used for that purpose. ${ }^{9}$

\section{RETRACTION INSTRUMENTS}

\section{Kim Trac Retractor}

Kim Trac retractors have more varying widths compared to other conventional retractors (they vary from $8 \mathrm{~mm}$ to $14 \mathrm{~mm}$ in comparison to conventional one of $10 \mathrm{~mm}$ ). Kim Trac retractors 1 and 2 comprise wings that can separate the elevated soft tissue and the area of surgery. These elevators can be used with plastic protector which are advantageous since it ensures easy flap retraction along with high accessibility and visibility to the operating field. They are able to anchor against the cortical bony plate stably and precisely because of its serrated end. ${ }^{9}$

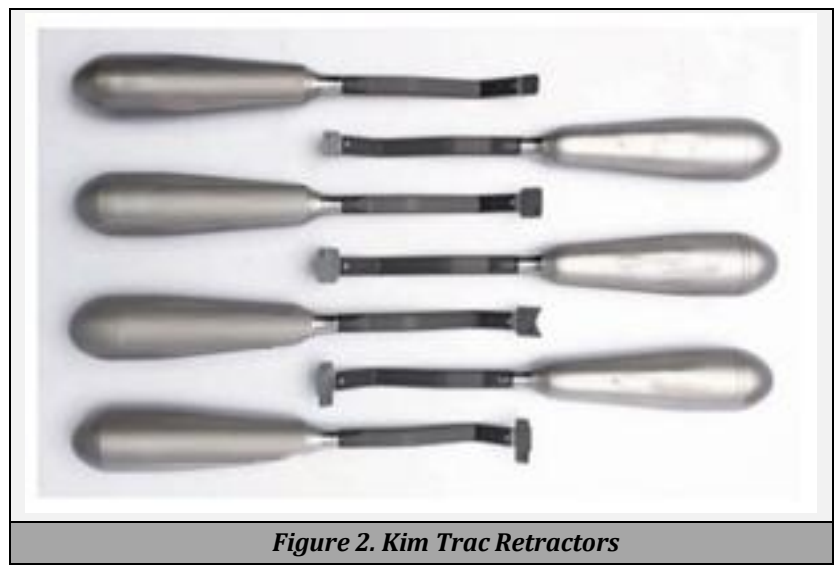

\section{Kim Pecora (KP) Retractors}

$\mathrm{KP} 1,2$, and 3 has wider tips in comparison to conventional retractors ( $15 \mathrm{~mm}$ to conventional $10 \mathrm{~mm}$ ) and are thinner by $0.5 \mathrm{~mm}$. The serrated ends of $\mathrm{KP}$ retractors anchor them securely on to the bone. Endodontic retractors are difficult to use on a convex or flat bone surface.

These tips are framed to the convexities and concavities of cortical bony plate. The comprehensive contact of the retractor tip to bone assures a stable and secure hold, thus, avoiding any sudden slippage which may result in swelling, traumatised tissue or painful healing. ${ }^{9}$ 


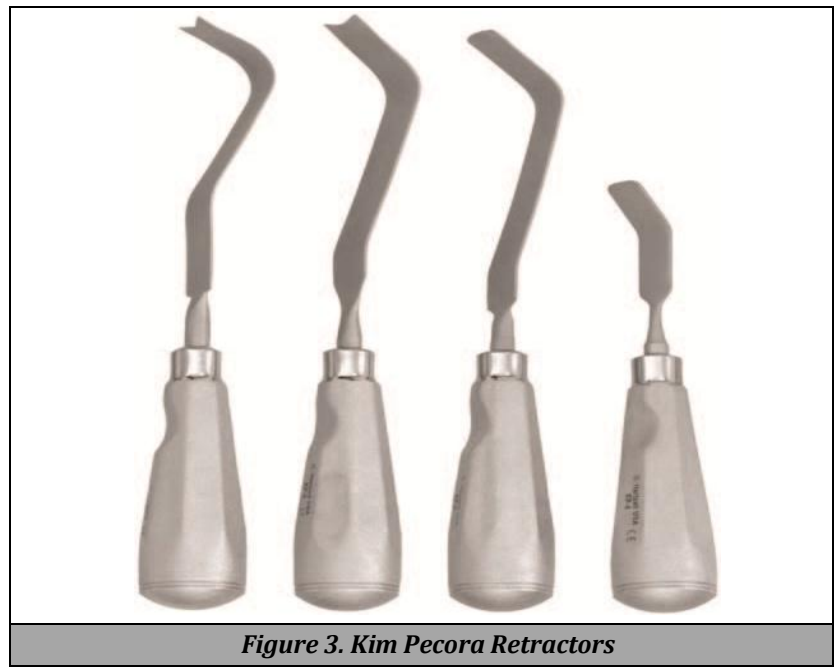

\section{Carr Retractors}

These retractors are $45^{\circ}$ angled and $90^{\circ}$ angled with serrated tips. A serrated tip reduces slippage of instrument and have ergonomic groove for thumb placement. ${ }^{5}$

\section{OSTEOTOMY AND ROOT END \\ RESECTION INSTRUMENT}

\section{$45^{\circ}$ Surgical Handpiece}

The $45^{\circ}$ handpieces are designed to direct water along the surface of the bur and the air is ejected through the back of the handpiece. This process helps in reducing any chance of pyemia or emphysema and also creates much less splatter compared to a conventional handpiece. The $45^{\circ}$ angled head of the handpiece makes it much easier to visualise difficult to reach areas. $^{9}$ The endodontic microsurgeon can also use this handpiece to gain better access to the apices of mandibular molars and maxillary, because this turbine is offset at 45 degrees. ${ }^{5}$ When a long shank surgical bur is utilised with operating microscope, it can be placed with high level of accuracy in the posterior regions. ${ }^{13}$

\section{Lindemann Bur}

They are designed mainly for aggressive cutting of bone structure (used for osteotomies). The bur is formed by a one piece tungsten carbide. Special geometry of this Bur allows very efficient cutting of hard / bone tissues while removing the debris. This bur have fewer flutes resulting in less clogging, reduced frictional heat and efficient cutting. 5,8

\section{Piezotomes}

The bio-minimalistic trends are transforming endodontic surgery. Piezo surgery achieves micrometric cuts that diminish cortical bone loss along with retention of root length. The greatest advantage of minimal invasive approach is due to the fact that the bone plate is conserved and replaced thus allowing maximum regenerative potential. ${ }^{18}$ They provide minimal procedural bone loss and precise osteotomy because of working tips have cutting width of $0.1-0.2 \mathrm{~mm}$. These designs are comparable to lasers but with precise depth control and minimum soft-tissues damage because of oscillation frequency range. ${ }^{18}$

\section{Lasers}

Bone-cutting Laser devices in dentistry are "Erbium-laser", "Neodymium-laser" and "Carbon-dioxide laser". Although osteotomies with lasers allow a precise osteotomy design with substantial less procedural bone loss compared to burs, one major drawback that still remains with laser devises is the complete lack of osteotomy depth control, soft-tissue preservation. ${ }^{19}$

\section{ROOT END PREPARATION INSTRUMENTS}

Ultrasonic tips have fundamentally changed apical surgery. ${ }^{20}$ The retro preparation is more precise, coaxial with the root canal using the ultrasonic tips and also the complete circumference is cleaner and the retro fill is also more precise. $9,18,9$

\section{Carr Tips}

Root end preparations were prepared using a miniature contra angle handpiece with small burs or a straight slow speed handpiece. However, coaxial root end preparation along the root canal is not possible. In addition, it caused frequent perforation in the lingual side of the root. Piezoelectric ultrasonic instrument is one of the most significant advancements in endodontic microsurgery for root end preparation. Stainless steel carr tips (CT 1 - 5) were the first ultrasonic tips for endodontic surgery in 1990. Surgical ultrasonic tips that were first designed by Dr. Garry Carr are known as Carr tips or CTs. They are $0.25 \mathrm{~mm}$ in diameter and approximately $1 / 10^{\text {th }}$ of a conventional handpiece in size.

The CT 1 and CT 5 tips are used mainly for mandibular anterior and maxillary teeth. The CT 2 and CT 3 are double angled to accommodate work in posterior teeth. $8,9,20$

\section{Kim Surgical Tips}

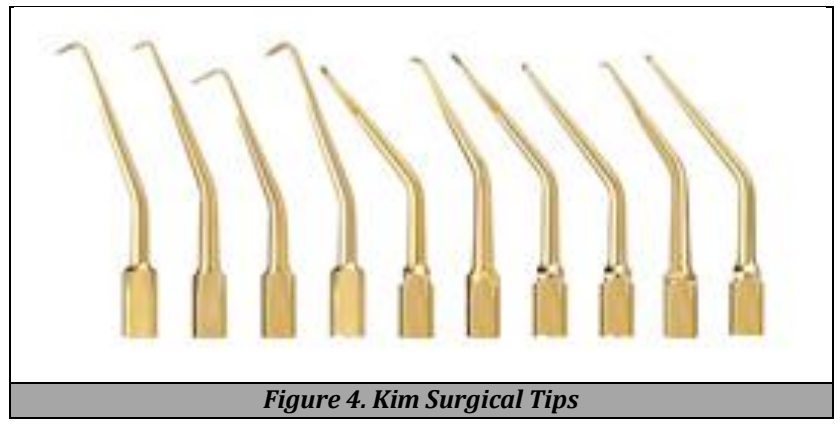

KiS (Kim Surgical) tips were introduced in 1999 by Spartan / Obtura. These ultrasonic tips are coated with zirconium nitride for a better cutting ability and have a more efficient irrigation port near the tip. They have a $3 \mathrm{~mm}$ cutting tip. These advanced tips help in efficient, smoother cutting and cause lesser microfractures due to the improved positioning of the irrigation port.8,9 The KiS tip 1 is designed for the premolars, mandibular anteriors whereas KiS 2 tip have a wider diameter 
used for maxillary anterior. The KiS3 tip has double bend, designed for posterior teeth (i.e., maxillary left side and the mandibular right side). The KiS 4 has tip angle of 110 degrees enabling it to reach the lingual apex of molar roots. The KiS 5 is used for maxillary right side and the mandibular left side. The KiS 4 tip counterpart is KiS 6 tip.

\section{JETips}

A special feature of these tips is that it has micro-projection at the cutting surfaces, allowing fast and complete removal of gutta-percha. The operator can bend the ultrasonic tip in any direction for better access. ${ }^{9}$

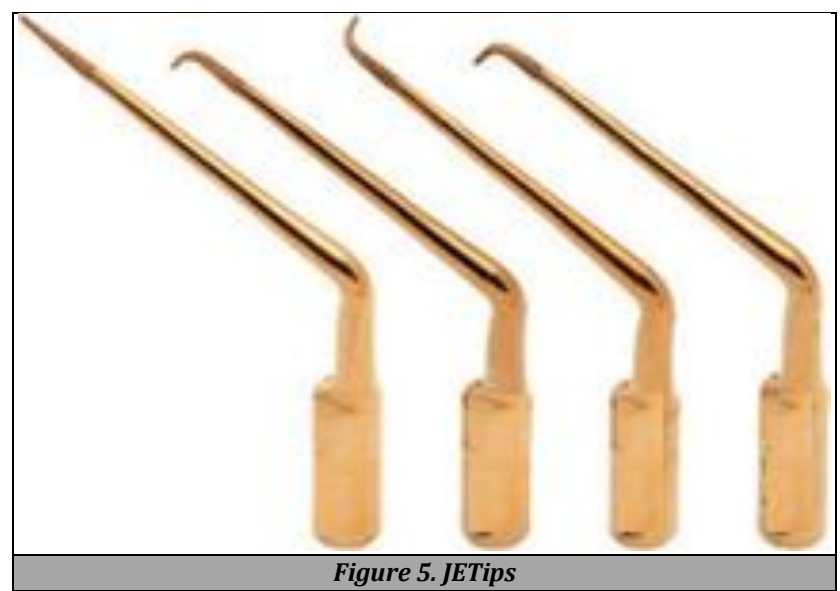

To achieve customised tip angle which is suitable for various micro-surgical requirements JETips are used which come in various sized tips ( 2 to $6 \mathrm{~mm}$ ) which facilitate bending with a jig used for bending the tip. The bending jig is configured to give flexibility to technicians to pick any angle and lengths which ensures that the microprojects are flawless without any damage during the bending process.

\section{Proultra Ultrasonic Tips}

ProUltra tips are precision instrument which are made of stainless steel that does not corrode irrespective of the irrigant used and does not chip or flake during intracanal operations which demand precision. This was achieved by applying an abrasive coat of zirconium nitrate to the stainless-steel ultrasonic tips to enhance the efficacy of the tips. Zirconumnitride coated tips are very efficient and provide good vision and leave a smooth dentin surface with microscopic ridges for bonding. ${ }^{21}$

\section{Diamond Coated Tips}

In retrograde cavity procedure the focus is to avoid cracking while removing dentin, this can be safely achieved by using the diamond coated tip as the sides of the tip is used instead of the chipping action utilised with instruments without a diamond coating. These tips are preferred for eliminating gutta percha in root end cavity. Diamond coated retro-tips abrade dentin more quickly and have been developed to minimise dentinal fractures. Therefore, the root-end cavity preparation time is shorter with diamond coated retro-tips. ${ }^{6,21,22}$

\section{Retrofill Carriers}

One can choose from a wide range of instruments for the placement of the retro-fill materials i.e., from an economical wipe-on block to a much more intricate and expensive micro apical placement (MAP) system.

\section{MAP System}

There have been many carriers, Dovgan carrier being the first carrier which had needles that would bend but was not a very efficient carrier for surgery. Dr Edward Lees block system which was introduced in 2000 which was effective during surgery but not very effective in clinical endodontics. Micro apical placement system was introduced which was a universal carrier with stainless steel applicator that had bayonet catch for exchangeable applicator cannulas. This bayonet catch was designed to hold various types of needles from a straight, curved to a triple angled one. This system can be used for both clinical and surgical endodontics. There were two types of needles for surgical procedure to ensure application of successive retro filling material in required portions. There types are the right angled and left angled needles, both with two external diameters $0.9 \mathrm{~mm}$ indicated in yellow and $1.1 \mathrm{~mm}$ indicated in red and two internal diameter $0.6 \mathrm{~mm}$ indicate din yellow and $0.8 \mathrm{~mm}$ indicated in red. ${ }^{23}$

\section{Teflon Sleeve and Plugger}

Teflon Sleeve and Plugger is a delivery instrument that has a tubing sleeve and a dispensing tip. The sleeve is made of inert tubing material like polyethylene, teflon etc. The sleeves inner diameter is almost the same as the dispensing tips outer diameter which allows the sleeve to be moved on the dispensing tip on application of pressure, even though there is a tight fit of the sleeve on the dispensing tip. ${ }^{24}$

\section{Microplugger and Condenser}

Microplugger and condenser are widely used for retro fill applications to compact filling material. They are instrument with two tips, one round and one oval. Round tip is used to create pot hole designs and oval tip is utilized to make isthmus designs. This was created in partnership with professor Abou Rass. ${ }^{14}$

\section{Microsurgical Condenser / Burnisher}

Microsurgical condenser is a very flexible tool which brings together a condenser and a ball burnisher on each end. Plugger comes in various sizes and also comes in two designs (left and right). ${ }^{14}$

\section{Irrigation Instruments \\ Stropko Irrigator, Microsuction}

Stropko Irrigator, Microsuction is a tool that uses a blunt micro tip (0.5 mm diameter) and is fitted on a standard water / air syringe. This is used for effective irrigation of retro preparation and to dry the preparation. 8,9 


\section{Suturing Instruments}

The suture materials should ideally have a surface that enables a traumatic passage through the tissues without generating capillarity so as to minimise immune response in the affected tissues. Microsurgical needles should have high flexural strength for them not to bend when passed through tough tissues. Moreover, they should be ductile enough to eliminate breakage when overloaded. ${ }^{9}$

To manage 5 - 0 or 6 - 0 synthetic sutures, Laschal microscissors along with the Castroviejo needle holder are used. The more delicate and smaller Castroviejo needle holder will require few adjustments in the beginning however will benefit with greater ease in difficult and delicate suturing. Earlier, the standard for endodontic surgery was 4 - 0 silk sutures, which are not recommended now a day. Plaque, food debris, and bacteria accumulate as the silk sutures are braided and thick, which also results in inflammation at the suture site. So as to eliminate these inflammations and any related delayed healing, monofilament sutures of nylon or polypropylene are used. ${ }^{9}$

- Laschal microscissors

- Castroviejo needle holder

- Micro-tissue forcep

\section{SUMMARY}

The objective of this article is to understand various microsurgical instruments that are utilised during microsurgical procedures. Conventional surgical instruments that are used are large for use in miniature surgical sites or traumatic to hard and soft tissues, hence, the advent of microsurgical instruments, designed for endodontic microsurgery. ${ }^{3}$ Instruments used in endodontic microsurgery are same as the ones used in conventional surgery, except that they are finer and smaller.10 Micro-scalpels are thus used for incisions, mainly in delicate areas such as the interdental papilla. Atraumatic flap elevation is then achieved using small, sharp, microsurgical periosteal elevators under dental operating microscope. Retractors comes in a range of serrated surfaces which are notched or flat to suit varying anatomical areas. They are designed to retract the flap and to keep the operators hand out of the microscope view. Design of Ultrasonic tips is such that it enables to prepare root-end cavities. Micro-mirrors make it convenient to examine the apical preparation ${ }^{3}$. In combination with the microscope, ultrasonic instrument allows conservative, coaxial root-end preparations and accurate root-end fillings which complies with the mechanical and biological requirements. ${ }^{12}$ Microsurgical suturing methods which involve smaller gauged tapered needles and smaller sizes sutures will also need utilisation of smaller micro-surgical tissue forceps, needle holders and scissors. The micro apical placement system or the Dovgan mineral trioxide aggregate (MTA) carrier permits precise placement of retrograde fillings, like MTA into the root-end cavity preparation without any spillage to the bony crypt. ${ }^{3}$ Micro surgical instruments have been developed over the years to take all the advantages of the increased visibility that is achieved from operating microscopes. ${ }^{10}$ Without microsurgical instruments, inspecting the root end, better visualisation of the surgical site have limited value.10
Predictable results in healing of lesions of endodontic origin is achieved by using microsurgical instruments and materials, matching biological concepts along with microsurgical approaches. ${ }^{10}$

\section{CONCLUSIONS}

The preservation of natural dentition must be the ultimate goal. Endodontic microsurgery has emerged as predictable treatment option which can be performed with accuracy with advanced microsurgical instrument and magnification, giving an option to the tooth that earlier used to be considered for extraction. Surgical instruments as per requirements can now be ordered from the catalogues provided by various manufactures. The way endodontic microsurgery is performed today has completely transformed mainly due to the integration of new technologies, techniques, and use of biomimetic materials

Financial or other competing interests: None.

Disclosure forms provided by the authors are available with the full text of this article at jemds.com.

\section{REFERENCES}

[1] Castellucci AR. Advances in surgical endodontics. L'informatore Endodontico 2003;6(1):2-16.

[2] Eliyas S, Vere J, Ali Z, et al. Micro-surgical endodontics. Br Dent J 2014;216(4):169-77.

[3] Seedat HC, Van der Vyver PJ, De Wet FA. Microendodontic surgery-part 1: surgical rationale and modern techniques. S Afr Dent J 2018;73(3):146-53.

[4] Pecora GE, Pecora CN. A new dimension in endo surgery: micro endo surgery. J Conserv Dent 2015;18(1):7-14.

[5] Chandra S, Gopikrishna V. Grossman's endodontic practice. 13 $3^{\text {th }}$ edn. Wolters Kluwer India Pvt Ltd., 2014.

[6] Kim S, Kratchman S. Modern endodontic surgery concepts and practice: a review. J Endod 2006;32(7):601-23.

[7] Endodontics AR. Endodontics colleagues for excellence. Chicago. Illinois: American Association of Endodontists 2013: p. 1-8.

[8] Ananad S, Soujanya E, Raju A, et al. Endodontic microsurgery: an overview. Dentistry Medical Research 2015;3(2):31-7.

[9] Kim S, Kratchman S, Karabucak B, et al. Microsurgery in endodontics. New Jersey: John Wiley \& Sons 2017.

[10] Dhingra S, Gundappa M, Bansal R, et al. Recent concepts in endodontic microsurgery: a review. TMU J Dent 2014;1(3)

[11] Pecora GE, Pecora CN. A new dimension in endo surgery: micro endo surgery. J Conserv Dent 2015;18(1):7-14.

[12] Jadun S, Monaghan L, Darcey J. Endodontic microsurgery. Part 2: armamentarium and technique. $\mathrm{Br}$ Dent J 2019;227(2):101-11.

[13] Carr GB, Murgel CAF. The use of the operating microscope in endodontics. Dent Clin North Am 2010;54(2):191-214.

[14] https://www.hufriedy.com/sites/default/files/308_Abo uRass_Microsurgical_Brochure_v18.pdf 
[15] https://www.hufriedy.com/surgical/periosteals/buser periosteal - elevator - black - line

[16] https://www.pearsondental.com/catalog/product.asp? majcatid $=948$

[17] Merino EM. Endodontic microsurgery for molars. In: Peters 0, edr. The Guidebook to Molar Endodontics. Berlin, Heidelberg: Springer 2017.

[18] Mohamed N, Nahmias Y, Serota K. The cortical window. Part 1: the evolution of endodontic surgery. Oral Health May 2018;108(5):60-6.

[19] Troedhan A, Mahmoud ZT, Wainwright M, et al. Cutting bone with drills, burs, lasers and piezotomes: a comprehensive systematic review and recommendations for the clinician. Int J Oral Craniofac Sci 2017;3(2):20-33.
[20] Niemczyk SP. Essentials of endodontic microsurgery. Dent Clin North Am 2010;54(2):375-99.

[21] İriboz E, Öztürk BA, Tarçin B. Advances and new approaches in endodontic surgery-a review. Paripex Indian Journal of Research 2015;4(4):4-10.

[22] Gunes B, Aydinbelge HA. Effects of ultrasonic root-end cavity preparation with different surgical-tips and at different power-settings on glucose-leakage of root-end filling material. J Conserv Dent 2014;17(5):476-80.

[23] Castellucci A, Papaleoni M. The MAP system: a perfect carrier for MTA in clinical and surgical endodontics. Roots 2009;3:18-22.

[24] Lemon RR, Luebke RG. Dental carrier device for dispensing slurry-like filling materials. United States 2001. 